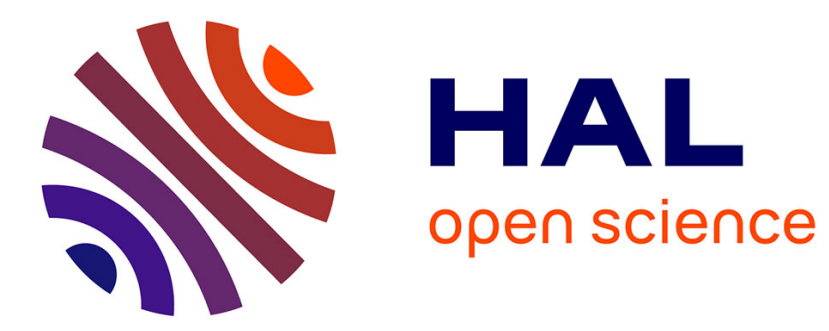

\title{
A formula for the power in partially failed PV fields
} G. Ambrosone, S. Catalanotti, U. Coscia, G. Troise

\section{To cite this version:}

G. Ambrosone, S. Catalanotti, U. Coscia, G. Troise. A formula for the power in partially failed PV fields. Revue de Physique Appliquée, 1983, 18 (10), pp.645-649. 10.1051/rphysap:019830018010064500 . jpa-00245126

\section{HAL Id: jpa-00245126 https://hal.science/jpa-00245126}

Submitted on 1 Jan 1983

HAL is a multi-disciplinary open access archive for the deposit and dissemination of scientific research documents, whether they are published or not. The documents may come from teaching and research institutions in France or abroad, or from public or private research centers.
L'archive ouverte pluridisciplinaire HAL, est destinée au dépôt et à la diffusion de documents scientifiques de niveau recherche, publiés ou non, émanant des établissements d'enseignement et de recherche français ou étrangers, des laboratoires publics ou privés. 


\title{
A formula for the power in partially failed PV fields
}

\author{
G. Ambrosone, S. Catalanotti, U. Coscia and G. Troise \\ Istituto di Fisica, Facoltà di Ingegneria, Università di Napoli, Piazzale Tecchio, Napoli, Italy
}

(Reçu le 10 février 1983, révisé le 30 juin, accepté le 7 juillet 1983)

\begin{abstract}
Résumé. - Dans cet article nous présentons des formules analytiques donnant les courbes de puissance en fonction de cellules endommagées pour quelques configurations. Les configurations analysées sont simples (au maximum elles sont constituées par quatre niveaux de connexion) car les premiers niveaux sont les plus responsables de la perte en puissance.
\end{abstract}

\begin{abstract}
In this paper we present analytical formulae which give the curves of power as a function of failed cells, for some configurations. The configurations analysed are simple (maximum four connection levels) because the first levels are the more responsible for the power decrease.
\end{abstract}

\section{Introduction.}

In previous papers $[1,2]$ we have studied the decrease of the power supplied by a photovoltaic field with failed cells. In the first of these papers [1] a probabilistic approach has been used; it gives exact analytical formulae which are difficult to use for large fields. In the second paper [2] the same problem has been treated with a statistical approach; in this case we have shown, for several configurations of the field, the power decrease as a function of the failure number. Both these methods require long computer calculations and therefore cannot be used for field configuration optimizations.

In this paper we want to overcome this obstacle by introducing an analytical expression for the power decrease as a function of the failure number and of the parameters of the field. This expression can be easily used to study the optimal configuration taking into account various problems such as the failure one, the connection costs, and so on.

Our analytical formula for the power function, in spite of the simplifications made, is sensibly accurate.

In this paper our hypotheses on the cell characteristics and on the failures are not described as they were widely discussed in reference 1 . We only study ideal cells with fill factor equal to one and the failures, mutually exclusive and statistically independent, produce a circuit opening inside the failed cell.

In the first section we briefly discuss the formalism and the power computation used in the paper. In the second section we present an analytical expression for the power as a function of the failure number in PS fields. In section 3 we expand this formula to more complex fields.

Finally, in section 4, we present a simple application of our formulae, to determine the optimal configuration of a photovoltaic field.

\section{The power calculation.}

This section is devoted to define some quantities and to give some more details about our problem.

Let us consider $T$ cells; the symbol $s_{1} p_{1} \ldots s_{n} p_{n}$ represents, in this paper, a field in which $s_{1}$ cells are series connected to constitute a group; $p_{1}$ groups are connected in parallel; $s_{2}$ sub-arrays $s_{1} p_{1}$ are connected in series, etc. Finally $p_{n}$ sub-arrays $s_{1} p_{1} s_{2} \ldots s_{n}$ are connected in parallel.

Obviously we can write

$$
\begin{aligned}
& T=\prod_{i=1}^{n} s_{i} p_{i}=s p \\
& s=\prod_{i=1}^{n} s_{i} \\
& p=\prod_{i=1}^{n} p_{i} .
\end{aligned}
$$

The current $I(0)$ supplied by the intact field is

$$
I(0)=I_{1} p
$$

where $I_{1}$ is the current produced by a single cell.

If there are $R$ failed cells, the current produced by the field will be proportional to $\left(1-R^{+}\right)$where 
$R^{+}$is the number of ways in which the current cannot flow. The fractional current will be, therefore

$$
I(R) / I(0)=1-R^{+} / p .
$$

Now we can consider fields connected to a power tracker, to a storage cell or to a resistive load. In the last case the power will be proportional to the square of the current whereas in the first two cases the power will be proportional to the current. We can conclude that the ratio :

$$
1-R^{+} / p
$$

will represent the fractional power for fields connected to a power tracker or to a storage cell or the square root of the fractional power for fields connected to a resistive load. If we are mainly interested in the slope of the power curves, we can treat the ratio as the fractional power; it is obvious that, if the load is resistive, the values given by our formulae must be squared. We can conclude, with this exception, that the fractional power is

$$
W(R) / W(0)=1-R^{+} / p .
$$

The power depends on the failure distribution, so we must determine the mean value of the fractional power, over all the possible distributions, to obtain the mean fractional power (m.f.p.)

$$
\bar{w}^{+}(R)=\bar{W}(R) / W(0)=1-\bar{R}^{+} / p .
$$

If we introduce the cell failure ratio (c.f.r.)

$$
x=R / T
$$

we obtain

$$
\bar{w}(x)=1-\bar{R}^{+} / p .
$$

In a previous paper [2] we have shown that PS fields have a regular behaviour and, moreover, that (concerning the failure problem) PS fields and the more complex ones (for instance SPS, PSP, SPSP) are very similar. In this paper we will use these results to obtain an analytical expression for the power. We do not treat SP fields; we only recall that for SP fields it is possible to find the exact formula, which can be easily simplified by using the probabilistic techniques.

\section{Analytical form of the m.f.p. in PS fields.}

From the considerations of section 1, we start to determine the analytical form of the m.f.p. for a PS field. In this case the m.f.p. is given by

$$
\bar{w}_{p s}\left(x_{p s}\right)=1-\bar{R}^{+} / p
$$

where $\bar{R}^{+}$is the maximum number of failed cells among the $s$ parallels.
Let us consider a $\mathrm{P}$ field, made up of $p$ parallel connected cells. The fractional power, when $R$ failures occur, will be

$$
w_{p}\left(x_{p}\right)=1-R / p=1-x_{p} .
$$

For a $\mathrm{P}$ field we can write

$$
\bar{w}_{p}\left(x_{p}\right)=w_{p}\left(x_{p}\right)
$$

i.e. the fractional power is independent of the specific distribution of the failures in the field.

Let us consider, now, a P2 field (i.e. a field built up with only two parallels). The c.f.r. $x_{p}$ and $x_{p 2}$ which supply the same m.f.p. are related by

$$
x_{p} / x_{p 2}=h(w, p) .
$$

Moreover, as it was stated in reference 2, values of c.f.r. $x_{p}, x_{p 2}, \ldots, x_{p 2^{n}}$ which give the same m.f.p. for $\mathrm{P}, \mathrm{P} 2, \ldots, \mathrm{P}^{n}$ fields must obey the following relations :

$$
x_{p} / x_{p 2}=x_{p 2} / x_{p 4}=\cdots=x_{p 2^{(n-1)} /} / x_{p 2^{n}}=h(w, p) .
$$

\section{Consequently}

$$
\begin{aligned}
& w_{p 2^{n}}\left(x_{p 2}\right)=w_{p}\left(x_{p}\right)=1-x_{p} \\
& x_{p} / x_{p 2^{n}}=\left[x_{p} / x_{p 2}\right]^{n}=[h(w, p)]^{n} .
\end{aligned}
$$

We will assume that, for any $s$

$$
\begin{gathered}
w_{p s}\left(x_{p s}\right)=w_{p}\left(x_{p}\right)=1-x_{p} \\
x_{p} / x_{p s}=\left(x_{p} / x_{p 2}\right)^{\log _{2} s}=[h(w, p)]^{\log _{2} s}
\end{gathered}
$$

then

$$
x_{p s}=x_{p} /[h(w, p)]^{\log _{2} s}
$$

To determine the function $h$ we have calculated the values of the ratios

$$
x_{p} / x_{p 2} ; \quad x_{p 2} / x_{p 4}, \ldots, x_{p 2^{n-1}} / x_{p 2^{n}}
$$

for fixed values of power $w_{p}$, with $p$ as parameter.

These ratios are function of the power $w_{p}$; as we can write

$$
w_{p}\left(x_{p}\right)=1-x_{p}=1-R_{p} / p,
$$

we can represent the ratios as a function of the failure number $R_{p}=p x_{p}$.

In figure 1 the ratios are plotted as a function of $R_{p}$ for four values of $p(p=3,10,20,50)$ and several values of $s$. As we can see, all the points can be considered as belonging to a single curve, when $R_{p} \ll p$. For $R_{p} \simeq p$ the curves are dependent on the $p$ value.

In equation 1 the function $h$ depends on two parameters $w$ and $p$; by a semitheoretical approach it is possible to deduce that these parameters are related so that the function $h$ depends only on the parameter $p(1-w)$. 


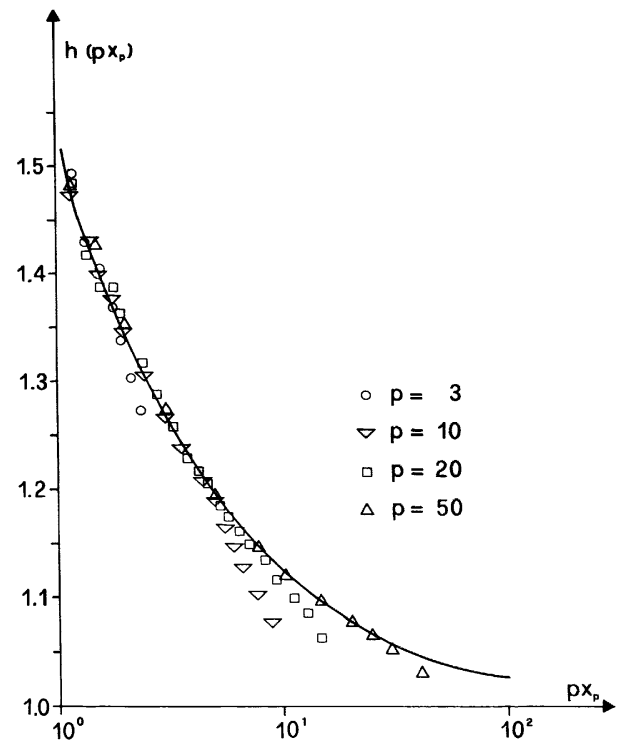

Fig. 1. - The ratios $x_{p s} / x_{p(2 s)}$ as a function of $p \cdot x_{p}$ for several values of $p$ and $s$. The curve represents the fit (with the corrective exponential term) specified in the text.

Let us consider a $\mathrm{P} 2$ field. For a fixed value of $R$, the possible values of $w_{p 2}^{+}(R)$ will correspond to specific values of failed cells pertaining to each of the parallels. Let us indicate by $R^{+}$the higher and by $R^{-}$the lower of these two values; we will write

$$
R^{+}=R / 2+d \text { and } R^{-}=R / 2-d
$$

where $d$ represents the fluctuation of the failures occurring in two parallels. The corresponding value of the fractionäl power will be

$$
w_{p 2}^{+}(R)=1-R^{+} / p
$$

and furthermore

$$
\bar{w}_{p 2}^{+}(R)=1-\bar{R}^{+} / p
$$

where

$$
\bar{R}^{+}=R / 2+\bar{d}
$$

The mean value (over all the possible distributions of $R$ failures in the P2 field) of the fluctuations, $\bar{d}$, will depend, of course, on $R$ and $p$. Now we remark that, if the failure number $R$ is much lower than $2 p$, the $(R+1)$-th failure can occur with identical probability in each of the two parallels, independently of the failures already occurred in each parallel. Therefore we can write

$$
\bar{d}=g(R) .
$$

We emphasize that this formula is valid only for $R \ll 2 p$.

By using this hypothesis, equation 2 becomes

$$
\bar{R}^{+}=R / 2+g(R)=[1+2 g(R)] R / 2=G(R) .
$$

For physical reasons the function $G(R)$ must be invertible and therefore we can write

$$
R=G^{-1}\left(\bar{R}^{+}\right) .
$$

Finally we obtain

$$
\begin{aligned}
R=\frac{2 \bar{R}^{+}}{1+2 g(R)} & =2 \bar{R}^{+} /\left\{1+2 g\left[G^{-1}\left(\bar{R}^{+}\right)\right]\right\} \\
& =2 \bar{R}^{+} / h\left(\bar{R}^{+}\right)
\end{aligned}
$$

i.e.

$$
x_{p 2}=\frac{R}{2 p}=\left(\bar{R}^{+} / p\right) / h\left(p \cdot \bar{R}^{+} / p\right)=\bar{x}_{p}^{+} / h\left(p \cdot \bar{x}_{p}^{+}\right)
$$

but $\bar{x}_{p}^{+}=x_{p}$ and therefore

$$
x_{p 2}=x_{p} / h\left(p \cdot x_{p}\right) .
$$

With this analysis we have obtained the same formula as in equation 1 . Here the dependence on $p$ and $x_{p}$ is specified as a dependence on $p . x_{p}$.

Now we can use the results shown in reference 2 to give the explicit form of the function $h\left(p \cdot x_{p}\right)$.

As we can see in figure 1 , the function $h$ appears to be a hyperbola. Therefore we use the function

$$
h\left(p \cdot x_{p}\right)=a+k /\left(b+p \cdot x_{p}\right) .
$$

The values" of the parameters which give the best fit for all the points, are

$$
\begin{aligned}
& a=1.0263 \\
& b=1.2502 \\
& k=1.0806 .
\end{aligned}
$$

We remark that our fit does not give a good agreement for values of $R_{p}$ near 1 , when $p$ is small, i.e. when the first failure causes a great decrease of the power. This region can be fitted by adding an exponential term; we obtain a new function

$$
h^{\prime}\left(p \cdot x_{p}\right)=a+k /\left(b+p \cdot x_{p}\right)+c \exp \left(-\tau p \cdot x_{p}\right)
$$

where $c=4.129 \times 10^{13}$ and $\tau=32.058$.

Once the explicit form of the function $h$ is known, we can compute the c.f.r. as a function of the m.f.p. value. We have

$$
\begin{aligned}
& x_{p s}\left(w_{p s}\right)=\left(1-w_{p s}\right) /\left\{h\left[p\left(1-w_{p s}\right)\right]\right\}^{\log s} \\
& 0 \leqslant w_{p s} \leqslant 1-1 / p .
\end{aligned}
$$

In figure 2 we compare the power curve calculated with our formula to the exact results presented in reference 2. As expected, the agreement is very good for high values of the m.f.p., for any $p$ and $s$. The error increases when the m.f.p. decreases because, in this case, $R_{p} \simeq p$ and, as previously said, our formulae are justified only if $R_{p} \ll p$. 


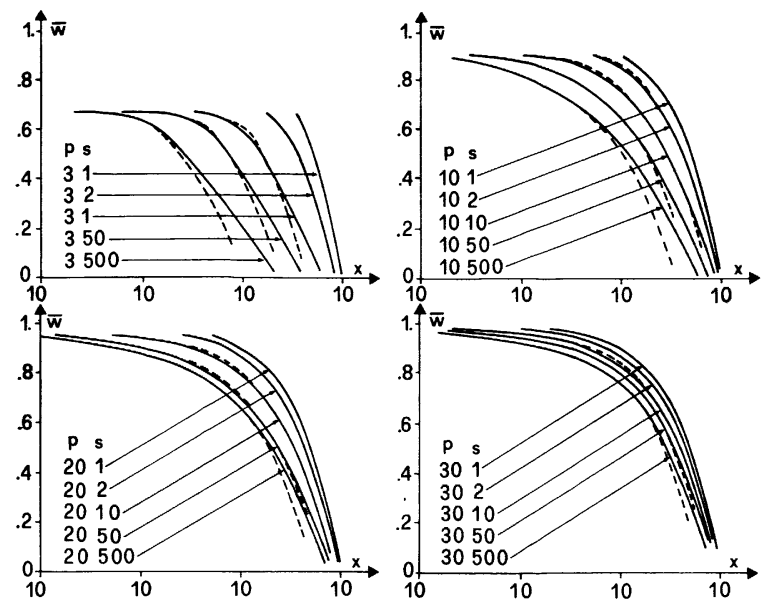

Fig. 2. - Power curves for several couples $p, s$. The full line curves represent the m.f.p. computed in reference 2 ; the dashed line curves represent the fit presented in the text.

\section{The analytical form of the m.f.p. for more complex fields.}

In this section we want to give the analytical formula of the m.f.p. for fields whose behaviour can be easily deduced from that of PS fields. As shown in reference 2 these fields are the PSP, the SPS and the SPSP field.

3.1 PSP FIELDS. - By using simple considerations (see Ref. 2) for a $p_{1} s_{1} p_{2}$ fields we can write

$$
\begin{array}{lcl} 
& w_{p_{1} s_{1} p_{2}}\left(x_{p_{1} s_{1} p_{2}}\right)=w_{p_{1} s_{1}}\left(x_{p_{1} s_{1}}\right) \\
\text { if } & x_{p_{1} s_{1} p_{2}}=x_{p_{1} s_{1}} \\
\text { when } & x_{p_{1} s_{1}}>1 /\left(p_{1} s_{1}\right) .
\end{array}
$$

Consequently we obtain

$$
w_{p_{1} s_{1} p_{2}}\left(x_{p_{1} s_{1} p_{2}}\right)=w_{p_{1} s_{1}}\left(x_{p_{1} s_{1}}\right)=w_{p_{1}}\left(x_{p_{1}}\right)=1-x_{p_{1}}
$$

if

or

$$
x_{p_{1} s_{1} p_{2}}=x_{p_{1}} /\left[h\left(p_{1} x_{p_{1}}\right)\right]^{\log _{2} s_{1}}
$$

$$
\begin{aligned}
x_{p_{1} s_{1} p_{2}}\left(w_{p_{1} s_{1} p_{2}}\right) & = \\
= & \left(1-w_{p_{1} s_{1} p_{2}}\right) /\left\{h\left[p_{1}\left(1-w_{p_{1} s_{1} p_{2}}\right)\right]\right\}^{\log _{2} s_{1}} \\
\text { for } \quad 0 & \leqslant w_{p_{1} s_{1} p_{2}} \leqslant 1-1 /\left(p_{1} p_{2}\right) .
\end{aligned}
$$

In this case the current decrease caused by the first failure is generally low, therefore in the explicit formula of the function $h$ the corrective exponential term is not needed.

3. 2 SPS FIELDS. - In reference 2 we showed that in a $s_{1} p_{1} s_{2}$ field the first series is not relevant, i.e. we can write

$$
w_{s_{1} p_{1} s_{2}}\left[R /\left(p_{1} s_{2}\right)\right]=w_{s_{1}^{\prime} p_{1} s_{2}}\left[R /\left(p_{1} s_{2}\right)\right]
$$

if

$$
s_{1} x_{s_{1} p_{1} s_{2}}=s_{1}^{\prime} x_{s_{1}^{\prime} p_{1} s_{2}} .
$$

This relation allows us to write

$$
\begin{aligned}
& w_{s_{1} p_{1} s_{2}}\left(s_{1} x_{s_{1} p_{1} s_{2}}\right)=w_{p_{1} s_{2}}\left(x_{p_{1} s_{2}}\right)=w_{p_{1}}\left(x_{p_{1}}\right)=1-x_{p_{1}} \\
& \text { if } \\
& \quad x_{s_{1} p_{1} s_{2}}=x_{p_{1}} /\left\{s_{1}\left[h\left(p_{1} x_{p_{1}}\right)\right]\right\}^{\log _{2} s_{2}} \\
& \text { or } \\
& x_{s_{1} p_{1} s_{2}}\left(w_{s_{1} p_{1} s_{2}}\right)= \\
& =\left(1-w_{s_{1} p_{1} s_{2}}\right) /\left\{s_{1}\left\{h\left[p_{1}\left(1-w_{s_{1} p_{1} s_{2}}\right)\right]\right\}^{\log _{2} s_{2}}\right\} \\
& 0 \leqslant w_{s_{1} p_{1} s_{2}} \leqslant 1-1 / p_{1} .
\end{aligned}
$$

3. 3 SPSP FIELDS. - The formula previously written can be used also for $s_{1} p_{1} s_{2} p_{2}$ fields because the m.f.p. is independent on the last parallel connection value $p_{2}$.

In this case we write

$$
w_{s_{1} p_{1} s_{2} p_{2}}\left(s_{1} x_{s_{1} p_{1} s_{2} p_{2}}\right)=w_{p_{1}}\left(x_{p_{1}}\right)=1-x_{p_{1}}
$$

for

$$
x_{s_{1} p_{1} s_{2} p_{2}}=x_{p_{1}} /\left\{s_{1}\left[h\left(p_{1} x_{p_{1}}\right)\right]\right\}^{\log _{2} s_{2}}
$$

or

$$
\begin{aligned}
& x_{s_{1} p_{1} s_{2} p_{2}}\left(w_{s_{1} p_{1} s_{2} p_{2}}\right)= \\
& \quad=\left(1-w_{s_{1} p_{1} s_{2} p_{2}}\right) /\left\{s_{1}\left\{h\left[p_{1}\left(1-w_{s_{1} p_{1} s_{2} p_{2}}\right)\right]\right\}^{\log _{2} s_{2}}\right\}
\end{aligned}
$$

for

$$
0 \leqslant w_{s_{1} p_{1} s_{2} p_{2}} \leqslant 1-1 /\left(p_{1} p_{2}\right)
$$

\section{Application example.}

To show the utility of our formulae for the m.f.p. we can study a PS field.

Let us suppose that we repair the field only when the fractional power supplied by the field decreases below 0.8 .

We want to determine the maintenance cost as a function of the number $p$ of parallel connected cells.

We suppose the cells to have a mean life $\tau$. Then the c.f.r. as a function of time $t$ is

$$
x_{p s}(t)=1-\exp (-t / \tau)
$$

The time interval between two maintenance operations will be

$$
T=\tau \ln \left[1 /\left(1-x_{p s}\right)\right] .
$$

Let us suppose, now, that the maintenance cost depends on two parameters

$$
C_{\mathrm{M}}=a+b R
$$

where $a$ is a constant value.

$b$ is the substitution cost of the failed cells.

The annual cost of maintenance will be

$$
c_{\mathrm{M}}=C_{\mathrm{M}} / T=(a+b R) / T
$$


and therefore

$$
c_{\mathrm{M}}=\frac{a+b s p x_{p s}}{\tau \ln \frac{1}{1-x_{p s}}}
$$

and finally

$$
c_{1}=\frac{\tau}{a} c_{M}=\frac{1+\left(b s p x_{p s}\right) / a}{\ln \left[1 /\left(1-x_{p s}\right)\right]} .
$$

For a numerical evaluation we put $b s p=300 a$ and therefore we obtain, for $p s=100000$, the data plotted in figure 3.

The optimal value of $p$ can, then, be obtained considering also the other aspects of the problem, such as connections cost and safety problems. As these informations are out of the interest of this paper, we do not treat them.

\section{Conclusions.}

We have developed an analytical method that allows to evaluate, in a quite satisfactory way, power curves of complex fields as a function of the number of failed cells. The expressions we have obtained are

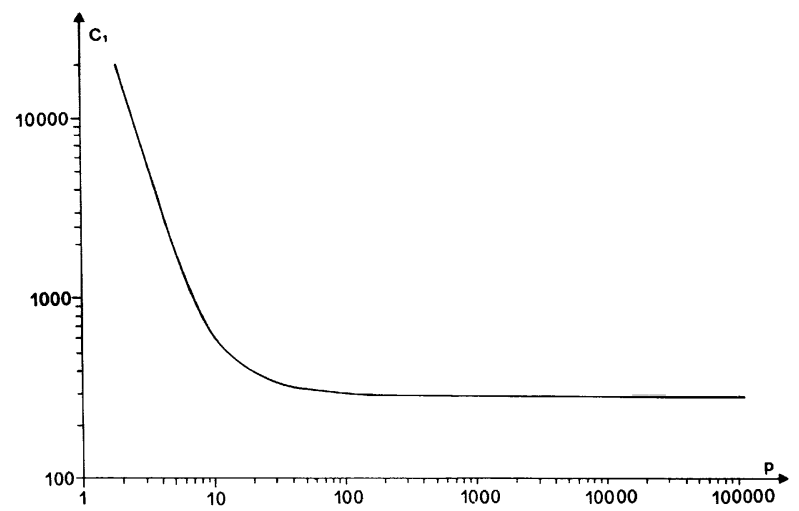

Fig. 3. - The equivalent maintenance $\operatorname{cost} c_{1}$ as a function of parallel number $p$, for PS fields.

valid when the number of failed cells is low. This corresponds to high values of fractional power, which is the situation of practical interest.

Our results enable us to say that the first connection levels are the more responsible for the power decrease and therefore they must be optimized with great care.

\section{References}

[1] Ambrosone, G. et al., Failure problem in photovoltaic fields : probabilistic approach (submitted for publication in Revue Phys. Appl.).
[2] Ambrosone, G. et al., A statistical solution for the failure problem in large photovoltaic fields (submitted for publication in Revue Phys. Appl.). 